\title{
(6) OPEN ACCESS \\ Monitoring the athlete training response: subjective self-reported measures trump commonly used objective measures: a systematic review
}

\author{
Anna E Saw, ${ }^{1}$ Luana C Main, ${ }^{2}$ Paul B Gastin ${ }^{1}$
}

- Additional material is published online only. To view please visit the journal online (http://dx.doi.org/10.1136/ bjsports-2015-094758).

${ }^{1}$ Centre for Exercise and Sport Science, Deakin University, School of Exercise and Nutrition Sciences, Burwood, Victoria, Australia

${ }^{2}$ Centre for Physical Activity and Nutrition Research, Deakin University, School of Exercise and Nutrition Sciences, Burwood, Victoria, Australia

\section{Correspondence to}

Anna E Saw, School of Exercise and Nutrition Sciences, Deakin University, 221 Burwood Highway, Burwood, VIC 3125 , Australia;

anna.saw@deakin.edu.au

Accepted 19 August 2015 Published Online First 9 September 2015

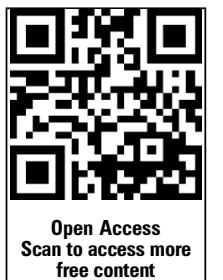

CrossMark

To cite: Saw AE, Main $\mathrm{LC}$, Gastin PB. Br J Sports Med 2016;50:281-291.

\section{ABSTRACT}

Background Monitoring athlete well-being is essential to guide training and to detect any progression towards negative health outcomes and associated poor performance. Objective (performance, physiological, biochemical) and subjective measures are all options for athlete monitoring.

Objective We systematically reviewed objective and subjective measures of athlete well-being. Objective measures, including those taken at rest (eg, blood markers, heart rate) and during exercise (eg, oxygen consumption, heart rate response), were compared against subjective measures (eg, mood, perceived stress). All measures were also evaluated for their response to acute and chronic training load.

Methods The databases Academic search complete, MEDLINE, PsycINFO, SPORTDiscus and PubMed were searched in May 2014. Fifty-six original studies reported concurrent subjective and objective measures of athlete well-being. The quality and strength of findings of each study were evaluated to determine overall levels of evidence.

Results Subjective and objective measures of athlete well-being generally did not correlate. Subjective measures reflected acute and chronic training loads with superior sensitivity and consistency than objective measures. Subjective well-being was typically impaired with an acute increase in training load, and also with chronic training, while an acute decrease in training load improved subjective well-being.

Summary This review provides further support for practitioners to use subjective measures to monitor changes in athlete well-being in response to training. Subjective measures may stand alone, or be incorporated into a mixed methods approach to athlete monitoring, as is current practice in many sport settings.

\section{INTRODUCTION}

Training imposes stress on an athlete, shifting their physical and psychological well-being along a continuum that progresses from acute fatigue to overreaching, and ultimately overtraining syndrome. ${ }^{1}{ }^{2}$ While overreaching may be carefully incorporated into a periodised training plan, progression towards overtraining syndrome is undesirable. Athletes should be closely monitored to ensure training elicits the desired effects on athlete well-being and performance. ${ }^{1-10}$

Performance, physiological, biochemical and subjective measures are all options for athlete monitoring. There is currently a relatively poor understanding of which measures are most appropriate. ${ }^{2}$ Performance is the ultimate indicator of physical and psychological well-being and the athlete's readiness to compete, yet it is impractical to test athletes daily via performance tests. ${ }^{11}$

A considerable body of research has investigated potential physiological mechanisms underlying the progression towards overtraining syndrome. ${ }^{12-16}$ Hormonal, immune, inflammatory and haematological parameters along with cardiovascular responses have been proposed as markers of these mechanisms, however findings have been inconsistent. This has been attributed to factors such as intra-assay and interassay variability, intraindividual and interindividual variability, the influence of circadian and pulsatile rhythms, nutrition and hydration status, climate, psychosocial factors and particular exercise characteristics. ${ }^{12}{ }^{14} \quad{ }^{17-19}$ Whether markers are elevated or depressed may also depend on the position along the athlete wellbeing continuum, with proposed physiological mechanisms involving an initial heightened response that later becomes exhausted. ${ }^{13-16}$

While there remains debate on the specific physiological mechanisms underlying the progression towards overtraining syndrome, there is agreement that progression is associated with psychological signs such as mood disturbances and symptoms similar to clinical depression. ${ }^{5} 20$ These signs and symptoms may be self-reported by athletes as perceived physical and psychological well-being, collectively termed 'subjective measures'. Subjective measures for routine athlete monitoring are also relatively cheap and simple to implement compared to objective measures. However, it is unknown whether subjective measures accurately reflect changes in athlete wellbeing, and how they can be effectively integrated into applied practice. $^{3}$

Therefore, we systematically reviewed whether subjective measures accurately reflected changes in athlete well-being (as objectively measured by performance, physiological and biochemical indicators) and whether subjective measures were responsive to acute changes in training load, and chronic training.

\section{METHODS}

\section{Search strategy}

An electronic search was conducted using the Academic search complete, MEDLINE, PsycINFO, SPORTDiscus and PubMed databases. The complete search strategy is presented in table 1 . Databases were searched from the earliest available record up to 5 May 2014, with results limited to humans and English language. 
Table 1 Database search strategy

\begin{tabular}{|c|c|}
\hline 1. Athlete & 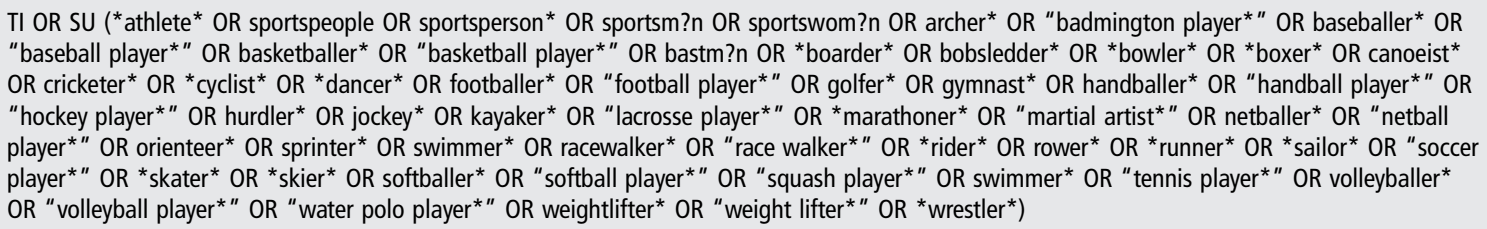 \\
\hline 2. Subjective measure & $\begin{array}{l}\text { SU OR AB (self-report* OR "self report" OR diary OR diaries OR questionnaire* OR survey* OR scale OR scales OR journal OR journals OR inventory } \\
\text { OR inventories OR self-evaluation OR "self evaluation" OR self-appraisal OR "self appraisal" OR self-assessment OR "self assessment" OR } \\
\text { self-rating OR "self rating" OR subjective OR perceive* OR perceptual OR "profile of mood states" OR "daily analys?s of life demands for athletes") }\end{array}$ \\
\hline 3. Objective measure & $\begin{array}{l}\text { SU OR AB (blood OR saliva* OR urine OR sweat OR serum OR plasma OR biological OR biochemical OR hormon* OR endocrine OR inflammat* OR } \\
\text { immune OR immunological OR cytokine OR haemato* OR physiological OR "heart rate" OR performance OR psychomotor OR neuromuscular OR } \\
\text { metaboli* OR "oxygen consumption" OR VO2* OR threshold OR "sleep actigraph*" OR electrocardiogra* OR ECG OR electromyogra* OR EMG OR } \\
\text { electroencephalogra* OR EEG OR "rate of force development") }\end{array}$ \\
\hline \multirow[t]{2}{*}{ 4. Athlete well-being } & 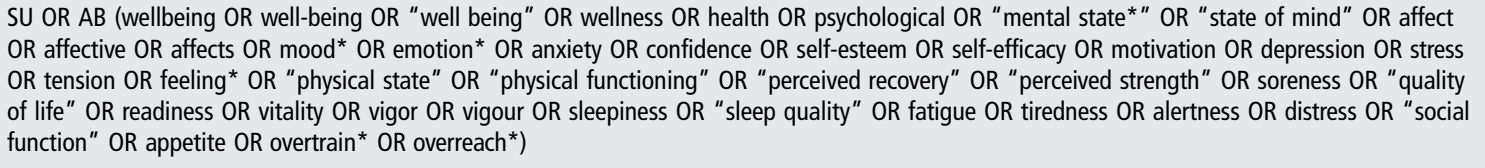 \\
\hline & 1 AND 2 AND 3 AND 4 \\
\hline
\end{tabular}

\section{Study selection}

Results were initially screened by title and abstract against the selection criteria. If it was unclear from the title or abstract whether a study met the inclusion criteria, the full-text was sought and screened against the selection criteria. The first author (AS) screened all articles.

Inclusion criteria:

A. Original research;

B. Study population of athlete(s) who were currently in training;

C. Not include a non-training experimental intervention;

D. Include a subjective self-report measure of athlete well-being with published validity and reliability;

E. Include an objective measure of athlete well-being; and

F. Have subjective and objective measures that coincide in timing, but not coincide with competition and be repeated at intervals reflective of the applied setting.

Studies that included a non-training experimental intervention such as nutritional supplementation or recovery strategy were excluded as the intervention may have confounded findings. Experimental manipulations of training (eg, overload, taper), were included as such manipulations are reflective of normal training periodisation and also enable evaluation of the sensitivity of subjective and objective measures to changes in training load.

Subjective measures were restricted to established self-report measures with validity and reliability reported in the literature to improve the credibility of findings and enable comparison between studies. Rating of perceived exertion was not included as a subjective measure as it is a measure of perceived exertion to a particular bout of exercise rather than a measure of athlete well-being.

Competition periods were excluded as they introduce acute perturbations to athlete well-being that are dependent on various individual and sport characteristics. ${ }^{21}$ Competitive performances are also influenced by external factors such as opponent and weather conditions that could affect athlete wellbeing and performance measures. The timing of administration of subjective and objective measures enabled the detection of concomitant change, while being practical for the applied setting (ie, excludes frequent measures across a day or measures taken during exercise).

\section{Data extraction}

The first author extracted data for all included studies using a specifically developed spreadsheet. Two authors (AS and PG) independently extracted data from five randomly selected studies. Discrepancies were resolved via adjudication by the third author (LM).

The relative weighting of study findings was determined by the magnitude of stimulus experienced by the athletes, and the magnitudes of change noted for subjective and objective measures. The magnitude of the stimulus influencing athlete wellbeing was rated as normal, moderate or high. Normal training stimuli included an ongoing training load without particular phases which may disrupt athlete well-being. Moderate training stimuli included overload and taper phases, while high-training stimuli included shock loads not reflective of normal practice that were designed to elicit considerable disruption to athlete well-being. Changes in subjective and objective measures across repeated measurements were noted if the study's analysis showed statistical significance or a meaningful effect size (ES) in a results table and/or text. For each measure, the magnitude of change (small $(\mathrm{p}=0.05, \mathrm{ES}=0.6)$, moderate $(\mathrm{p}<0.05, \mathrm{ES}<1.2)$, large $(p<0.01$, ES $>1.2))$, along with the direction of change, was noted.

Other data extracted from studies included the age, gender and participation level of athletes, sport characteristics, training phase and measures used. Measures of performance capacities were grouped as those measuring sustained performance (eg, time trial, submaximal workload) or short performance (eg, grip strength, vertical jump).

\section{Risk of bias assessment}

Studies were assessed for risk of bias to give a weighting to their contribution to the review. As no suitable published assessment criteria were available, specific criteria were developed (table 2). Scores were allocated based on how well each criterion was met, up to a maximum possible score of 8 (low risk of bias). Studies with a risk of bias assessment score of 4 or less were considered poor and their contribution to results was weighted as half.

\section{Data synthesis}

Meta-analysis was precluded due to heterogeneity in methods and reporting. Therefore, data were synthesised descriptively. 
Table 2 Risk of bias assessment criteria

\begin{tabular}{|c|c|c|c|c|c|}
\hline \multirow{2}{*}{\multicolumn{2}{|c|}{ Criteria }} & \multirow{3}{*}{$\begin{array}{l}\text { Definition } \\
\text { Study published in peer-reviewed journal }\end{array}$} & \multicolumn{3}{|c|}{ Scoring } \\
\hline & & & \multirow{2}{*}{$\begin{array}{l}0 \\
\text { No }\end{array}$} & \multirow{2}{*}{1} & \multirow[t]{2}{*}{2} \\
\hline A & Peer reviewed & & & & \\
\hline B & Number of participants* & Number of participants included in study findings & $<5$ & $5-50$ & $>50$ \\
\hline C & Population defined & Age, gender, sport, participation level and experience stated & No & Partly & Yes \\
\hline D & Training or competition load described & Training or competition undertaken during the study period is described & No & Partly & Yes \\
\hline $\mathrm{E}$ & Response set on self-report measure described & Response set (eg, 'right now' or 'in the past week including today') is described & No & Yes & \\
\hline
\end{tabular}

To determine the association between changes in subjective and objective measures, each association from an individual study was rated according to its direction (positive, negative, no association) and strength as determined from reported correlations (small $(\mathrm{r}<0.3)$, moderate $(\mathrm{r}=0.3-0.5)$, large $(\mathrm{r}>0.5))$ or average magnitudes of change. Individual associations were then summed and rated according to the predetermined levels of evidence outlined below (adapted from Van Tulder et $a l^{22}$ and Reurink et $a l^{23}$ ).

Strong evidence: consistently identified in two or more studies, and greater than or equal to $75 \%$ of all contributing findings.

Moderate evidence: consistently identified in two or more studies, and greater than $50 \%$ of all contributing findings.

Limited evidence: identified in one study, with greater than $50 \%$ of all contributing findings.

Conflicting evidence: inconsistency in two or more studies. If half of all contributing sources were in agreement, with the other half conflicting, a potential direction was indicated.

No evidence: no change in subjective or objective measures.

To determine the responsiveness of each subjective and objective measure to acute and chronic training load, the sensitivity and consistency of responses were evaluated. To evaluate the sensitivity of responses, the magnitude of change was multiplied by a factor weighting for the magnitude of stimulus (normal stimulus $\times 1$, moderate $\times 0.75$, high $\times 0.5$ ). In the instance of no change, factor weightings (normal $\times 1$, moderate $\times 1.5$ and high $\times 2$ ) were applied to reflect a lack of sensitivity. Individual changes were then summed to determine typical patterns, with the strength of evidence rated by the criteria above.

\section{RESULTS}

\section{Included studies and measures}

From an initial 4244 articles retrieved from database searches, 1377 were excluded as duplicates. A total of 2803 articles did not meet the inclusion criteria and were excluded (figure 1). Of the 64 remaining articles, 2 shared data with another article $^{24-27}$ and so were combined and considered as a single data set in each instance. A further six articles ${ }^{28-33}$ were excluded as the presentation or use of their data precluded interpretation of change across all athletes (eg, data used to retrospectively group athletes as overtrained or control). Finally, 56 studies were included in the analysis. Two of these studies were excluded from the responsiveness analysis as their primary stimulus was dietary restriction rather than training. ${ }^{34} 35$ Characteristics of included articles and results of the risk of bias assessment are provided in online supplementary table S1.

The most common subjective measures of athlete well-being were the Profile of Mood States (POMS) ${ }^{36}$ (including derivatives of the POMS ${ }^{37}$ ), Recovery Stress Questionnaire for Athletes $(\text { RESTQ-S })^{38}$ and Daily Analyses of Life Demands of Athletes (DALDA). ${ }^{39}$ Other measures included the overtraining questionnaire of the Societe Francaise de Medecine du Sport (SFMS), ${ }^{40}$ State-Trait Anxiety Inventory (STAI), ${ }^{41}$ Perceived Stress Scale (PSS), ${ }^{42}$ Multi-Component Training Distress Scale (MTDS), ${ }^{43}$ Competitive State Anxiety Inventory-2 (CSAI-2), ${ }^{44}$ Derogatis Symptom Checklist (DSC), ${ }^{45}$ State-Trait Personality Inventory $(\mathrm{STPI})^{46}$ and a mood questionnaire by Choi and Salmon ${ }^{47}$ (Mood).

\section{Associations between subjective and objective measures of athlete well-being}

There was moderate evidence for a negative association between stress and cortisol (table 3), and a positive association between vigour and leukocytes (table 4). There was moderate evidence for a positive association between four RESTQ-S stress subscales and creatine kinase $(\mathrm{CK})$, yet evidence was conflicting for total stress (table 4). Between the POMS and maximal oxygen consumption $\left(\mathrm{VO}_{2} \mathrm{max}\right)$, there was strong evidence for a positive association with the vigour subscale and moderate evidence for a negative association with the fatigue subscale, which was also reflected by strong evidence for a negative association with total mood disturbance (table 5). There was strong evidence for a negative association between symptoms of stress (measured by DALDA) and sustained performance (table 5).

\section{Responsiveness of subjective and objective measures to training}

Within studies (ie, under the same conditions), subjective measures were more sensitive and consistent than objective measures in 22 of the 54 studies. Objective measures were generally unresponsive to acute changes in training load, with moderate evidence of a typical response in three measures (CK, short and sustained performance) to both increased and decreased training (table 6). With ongoing training, there was moderate to strong evidence of responsiveness for five objective measures, including impairment of epinephrine/norepinephrine and leucocytes (table 6).

Subjective measures were more responsive to training than objective measures. There was moderate to strong evidence of impaired well-being with an acute increase in training load for 13 subjective measures (table 7). There was moderate to strong evidence of improved well-being with an acute increase in training load for 17 subjective measures (table 7). There was moderate to strong evidence of impaired well-being with ongoing training for 13 subjective measures (table 7). Six of the RESTQ-S measures (stress, fatigue, recovery, physical recovery, general well-being, being in shape) demonstrated moderate to strong evidence of responsiveness to all three of the training load conditions. Consolidation of subjective measures into a total score typically resulted in reduced sensitivity, with one in five studies reporting both subscale and total scores noting a change in only the subscale score(s). 


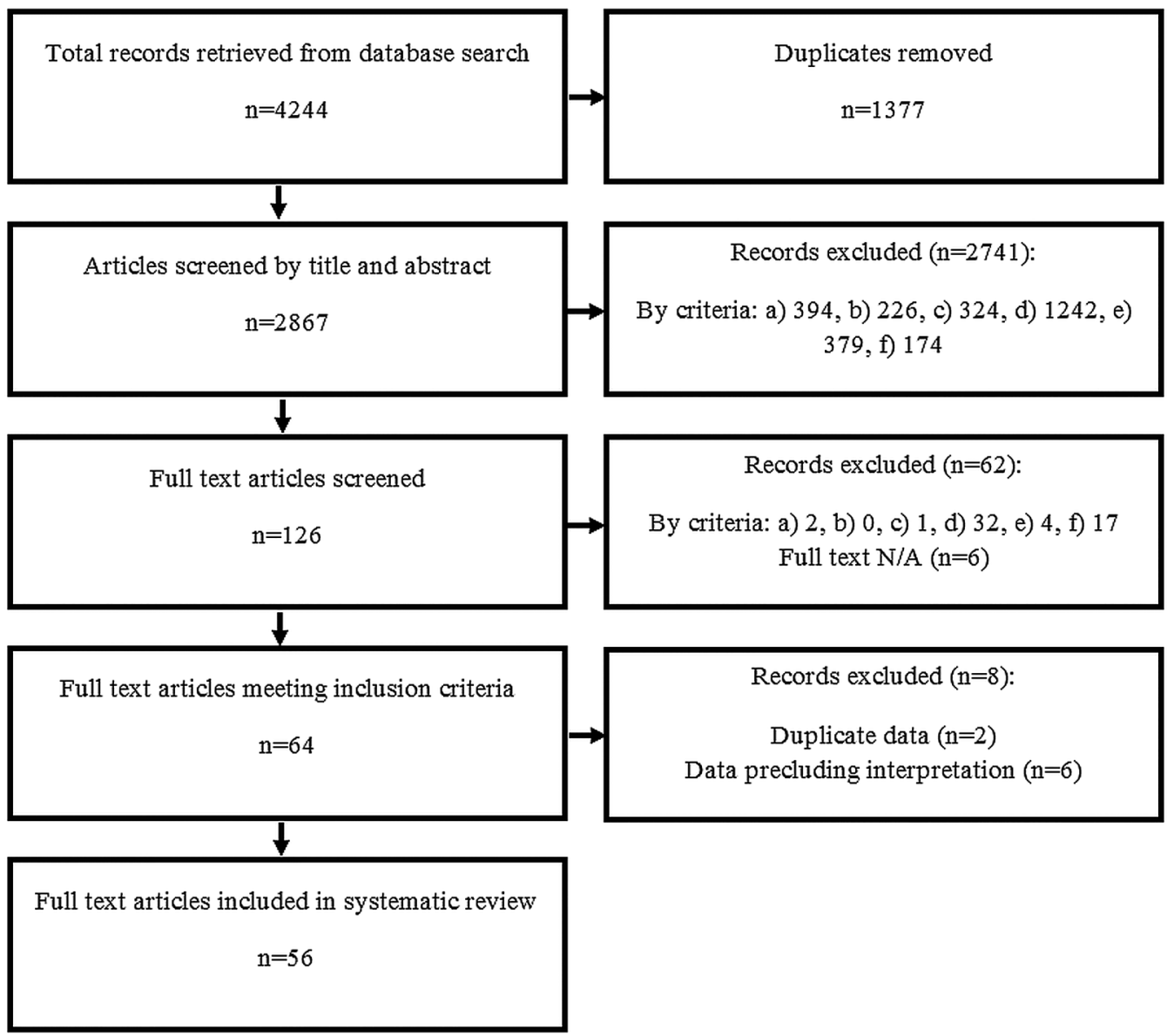

Figure 1 Selection of studies flow chart. Studies excluded for not meeting the following criteria: (A) original research; (B) study population of athlete(s); (C) no non-training intervention; (D) include a subjective measure; (E) include an objective measure; and (F) appropriate timing of measures.

\section{DISCUSSION}

This systematic review evaluated the ability of individual subjective measures, assessed by self-report measures, to reflect changes in athlete well-being. The findings provide support for subjective measures to reflect acute and chronic training-related changes in athlete well-being. In particular, measures of mood disturbance (eg, POMS), perceived stress and recovery (eg, RESTQ-S) and symptoms of stress (eg, DALDA) were widely investigated and appear to be useful for athlete monitoring.

\section{Subjective measures are responsive to acute and chronic training load}

Subjective measures consistently identified impaired well-being with acute increases in training load, ${ }^{26}$ 48-62 $^{-6}$ and improved well-being with an acute reduction in training load. ${ }^{26} 484952-58$ 60 63-67 This extends previous narrative reviews of overtraining markers that suggested subjective measures change in a doseresponse manner to training load. ${ }^{3} 78$ The ability of subjective measures to reflect both acute increases and decreases in training indicates that the measures are indeed responsive to training load. $^{68}$

Subjective measures also identified impaired well-being in response to chronic training load..$^{63} 64$ 69-78 This is particularly important for ongoing monitoring because progression towards overtraining syndrome may be gradual and less easily identified than in situations of acute overload. ${ }^{3}$ However, it is important to note that this finding does not take into account the different scenarios across studies, or the influence of non-training stressors on athlete well-being. Consequently, the tendency for well-being to be impaired with ongoing training should be interpreted cautiously as there was no way to discern where athletes may have been placed on the athlete well-being continuum.

\section{A case for subjective measures having some advantages over objective measures}

Subjective measures, particularly measures of mood disturbance, perceived stress and recovery and symptoms of stress, responded with superior sensitivity and consistency compared to objective measures. Within studies (ie, under the same conditions), sensitivity, consistency and/or timing differed in $46 \%$ of studies and $85 \%$ of these favoured subjective measures. Superior responsiveness of subjective measures over objective measures has been noted previously in experimental overload ${ }^{48} 55$ and observational $^{79}$ studies.

We found moderate evidence that creatine kinase increases and decreases with acute training load. Subjective well-being typically has the opposite response to acute loads, however only four subjective measures of stress (general stress, emotional stress, fatigue, emotional exhaustion) were moderately associated with creatine kinase. The lack of an association between creatine kinase and subjective measures may be explained by the different responses of these measures to chronic training. We found that creatine kinase was unresponsive to chronic training, likely due to minimal muscle damage induced by accustomed 
Table 3 Evidence of associations between subjective and objective (endocrine and erythrocytes) measures

\begin{tabular}{|c|c|c|c|c|c|c|c|c|c|c|c|c|c|}
\hline & \multicolumn{10}{|c|}{ Endocrine } & \multicolumn{3}{|c|}{ Erythrocytes } \\
\hline & C & $T$ & $\mathrm{~T}: \mathrm{C}$ & E/NE & Pro & GH & ACTH & LH & Dop & IGF-1 & RBC & Hct & $\mathrm{Hb}$ \\
\hline \multicolumn{14}{|l|}{ POMS } \\
\hline Total mood disturbance & $x x$ & $x x x$ & $x x x$ & $x x$ & $x$ & & & $x$ & $x$ & & - & - & - \\
\hline Tension & $x x x$ & $x x$ & $x x$ & $x x x$ & $x$ & & & $x$ & $x$ & - & - & - & - \\
\hline Depression & $x x$ & $x x$ & $x x$ & $x x x$ & $x$ & & & $\mathrm{x}$ & - & - & - & - & $?$ \\
\hline Anger & $x x$ & $x x$ & $x x x$ & $?$ & $x x x$ & & & $x$ & - & - & $?$ & $?$ & $x x x$ \\
\hline Confusion & $x x$ & $? \mathrm{x}$ & $x x$ & $x x x$ & $x$ & & & $x$ & $x$ & - & - & - & $?$ \\
\hline Vigour & $?$ & $x x$ & ?x & $?$ & - & & & - & $x$ & - & $x$ & $x$ & $?$ \\
\hline Fatigue & $x x$ & $x x$ & $x x x$ & $x x$ & $x$ & & & $x$ & $x$ & $x$ & - & - & $?$ \\
\hline \multicolumn{14}{|l|}{ RESTQ-S } \\
\hline Recovery-Stress Index & $x x x$ & $x x x$ & $\mathrm{x}$ & $x$ & $x$ & - & $\mathrm{x}$ & & & & - & $?$ & $?$ \\
\hline Stress & $\downarrow \downarrow$ & $x$ & $x$ & $x$ & $x$ & $x$ & $x$ & & & & - & $?$ & $?$ \\
\hline General stress & $x x$ & $x$ & - & $?$ & - & & - & & & & - & - & $?$ \\
\hline Emotional stress & $x x x$ & $x$ & - & $?$ & - & & - & & & & - & - & $?$ \\
\hline Social stress & $x x x$ & $\mathrm{x}$ & - & $?$ & - & & - & & & & $x$ & $?$ & $x \mathrm{xx}$ \\
\hline Conflicts/pressure & $?$ & $x$ & - & $?$ & - & & - & & & & - & - & $?$ \\
\hline Fatigue & $?$ & $\downarrow$ & - & $\uparrow$ & - & & - & & & & - & - & $?$ \\
\hline Lack of energy & ?x & $x x x$ & $x$ & $x$ & $x$ & & $x$ & & & & - & $?$ & $?$ \\
\hline Physical symptoms & $x x x$ & $?$ & $x$ & $x x x$ & $x$ & & $x$ & & & & - & $?$ & $?$ \\
\hline Injury & $x x x$ & $\downarrow$ & $x$ & $x$ & $x$ & & $x$ & & & & $?$ & ?x & $?$ \\
\hline Emotional exhaustion & $x x x$ & $x$ & - & $?$ & - & & - & & & & - & - & $?$ \\
\hline Disturbed breaks & $\mathrm{xx}$ & $x$ & - & $?$ & - & & - & & & & - & - & $?$ \\
\hline Recovery & $? x$ & $?$ & $x$ & $x$ & $x$ & $x$ & $x$ & & & & - & $?$ & $?$ \\
\hline Social recovery & $?$ & $\uparrow$ & - & $?$ & - & & - & & & & - & - & $?$ \\
\hline Physical recovery & $x x$ & $x x x$ & $x$ & $x$ & $x$ & & $x$ & & & & - & $?$ & $?$ \\
\hline General well-being & $x x$ & $x x x$ & $x$ & $x$ & $x$ & & $x$ & & & & - & $?$ & $?$ \\
\hline Sleep quality & $x x x$ & $\uparrow$ & - & $?$ & - & & - & & & & - & - & $?$ \\
\hline Success & $x x$ & $\uparrow$ & - & $?$ & - & & - & & & & - & - & $?$ \\
\hline Being in shape & $x x x$ & $?$ & $x$ & $x$ & $x$ & & $x$ & & & & $x$ & $x x x$ & $? x$ \\
\hline Personal accomplishment & $x x$ & $x$ & - & $?$ & - & & - & & & & - & - & $?$ \\
\hline Self-efficacy & $x x x$ & $\downarrow$ & - & $?$ & - & & - & & & & - & - & $?$ \\
\hline Self-regulation & $?$ & $x$ & - & $?$ & - & & - & & & & $?$ & - & $x x x$ \\
\hline \multicolumn{14}{|l|}{ SFMS } \\
\hline Overtraining score & $?$ & $?$ & $?$ & $x$ & & & & & & & & & \\
\hline \multicolumn{14}{|l|}{ DALDA } \\
\hline Sources of stress & - & & & - & & & & & - & & & & \\
\hline Symptoms of stress & $x$ & & & $\mathrm{x}$ & & & & & $x$ & & & & \\
\hline$n$ & 24 & 11 & 8 & 7 & 3 & 1 & 1 & 1 & 1 & 1 & 2 & 3 & 4 \\
\hline
\end{tabular}

Associations rated as positive ( $\uparrow$ ), negative ( $\downarrow)$, not associated (x), conflicting findings (?), no change (-). Three symbols indicates strong evidence, two symbols for moderate evidence, and one symbol for limited evidence. Shading highlights strong (dark grey) and moderate (light grey) associations. Abbreviations for objective measures: cortisol (C), testosterone (T), epinephrine and norepinephrine (E/NE), prolactin (Pro), growth hormone (GH), adrenocorticotropic hormone (ACTH), luteinising hormone (LH), dopamine (Dop), insulin-like growth factor-1 (IGF-1), erythrocytes (RBC), Haematocrit (Hct), haemoglobin (Hb). n refers to number of contributing studies.

POMS, Profile of Mood States; RESTQ-S, Recovery Stress Questionnaire for Athletes; SFMS, Societe Francaise de Medecine du Sport; DALDA, Daily Analyses of Life Demands of Athletes.

training. ${ }^{80}$ Chronic responses in the instance of overtraining are also inconsistent, questioning the utility of creatine kinase to monitor athlete well-being. ${ }^{78}$

We found moderate evidence that a reduction in acute training load was associated with an improvement in $\mathrm{VO}_{2}$ max. The noted positive association between $\mathrm{VO}_{2}$ max and subjective wellbeing may indicate that subjective measures reflect an athlete's ability to perform a sustained, maximal effort. Alternatively, subjective measures may reflect an athlete's psychological readiness to perform, with psychological state known to influence performance. ${ }^{81}$ Impaired $\mathrm{VO}_{2}$ max may also be attributable to reduced exercise time during the test rather than a decrement in physiological function. ${ }^{3}{ }^{82}$ Notably, a similar association with sustained performance was not found.
The lack of association between subjective and objective measures provides support for the inclusion of both in different yet complementary athlete monitoring roles.

While a lack of responsiveness is a limitation of objective measures for monitoring athlete well-being, their utility lies in measuring certain constructs which are related to athlete wellbeing, but do not necessarily reflect an athlete's position on the continuum. For instance, objective measures quantify physiological and performance capacities (eg, $\mathrm{VO}_{2} \mathrm{max}$, lactate threshold) which may be used to guide training prescription, and laboratory tests can detect medical conditions (eg, recurrent infection, ${ }^{83}$ iron deficiency ${ }^{84}$ ). 
Table 4 Evidence of associations between subjective and objective (immune, inflammation and muscle damage) measures

\begin{tabular}{|c|c|c|c|c|c|c|c|c|c|c|c|c|c|c|c|c|}
\hline & \multicolumn{3}{|c|}{ Immune } & \multicolumn{13}{|c|}{ Inflammation and muscle damage } \\
\hline & Leuk & $\lg A$ & $\lg G$ & IL-1B & IL-6 & IL-8 & IL-10 & IL-12p70 & TNF- $\alpha$ & $\alpha$-amylase & CRP & $\begin{array}{l}\text { Ox. } \\
\text { Stress }\end{array}$ & $\begin{array}{l}\text { GSH: } \\
\text { GSSG }\end{array}$ & Urea & Creatinine & CK \\
\hline \multicolumn{17}{|l|}{ POMS } \\
\hline Total mood disturbance & $x x x$ & $?$ & $x x x$ & & & & & & & & $x$ & $?$ & $x$ & $\mathrm{x}$ & $\mathrm{x}$ & $x x$ \\
\hline Tension & $x x x$ & $?$ & $\mathrm{x}$ & & $x$ & & & & & & $x$ & $?$ & $x$ & $x$ & $x$ & - \\
\hline Depression & $x x x$ & $?$ & $x$ & & $x$ & & & & & & $x$ & $?$ & $x$ & - & $x$ & $?$ \\
\hline Anger & $x x$ & $?$ & $\mathrm{x}$ & & $\mathrm{x}$ & & & & & & - & $?$ & $?$ & - & $\mathrm{x}$ & $?$ \\
\hline Confusion & $x x x$ & $?$ & $\mathrm{x}$ & & $\mathrm{x}$ & & & & & & $x$ & $?$ & $x$ & $x x$ & $x$ & - \\
\hline Vigour & $\uparrow \uparrow$ & $?$ & $?$ & & $\mathrm{x}$ & & & & & & $\uparrow$ & $x x x$ & $\downarrow$ & $x \mathrm{xx}$ & $\uparrow$ & $?$ \\
\hline Fatigue & $x x$ & $?$ & $?$ & & $\uparrow$ & & & & & & $x x x$ & $?$ & $x$ & $x x x$ & $\uparrow$ & $?$ \\
\hline \multicolumn{17}{|l|}{ RESTQ-S } \\
\hline Recovery-Stress Index & $x x x$ & & & & & & & & & & $x$ & - & $x$ & $\downarrow$ & $x$ & ?x \\
\hline Stress & $x x x$ & & & & & & & & & $\downarrow$ & $x$ & - & $x$ & $\uparrow$ & $x$ & $?$ \\
\hline General stress & $x x x$ & & & & & & & & & $\downarrow$ & $x$ & - & $x$ & $x$ & - & $\uparrow \uparrow$ \\
\hline Emotional stress & $x x x$ & & & & & & & & & $\mathrm{x}$ & $x$ & - & $x$ & $\mathrm{x}$ & - & $\uparrow \uparrow$ \\
\hline Social stress & $?$ & & & & & & & & & $x$ & $\uparrow$ & $x$ & $\downarrow$ & $x$ & - & $? \uparrow$ \\
\hline Conflicts/pressure & $x x x$ & & & & & & & & & $\downarrow$ & $x$ & - & $x$ & $x$ & - & $?$ \\
\hline Fatigue & $x x x$ & & & & & & & & & $\downarrow$ & $x$ & - & $x$ & $\mathrm{x}$ & - & $\uparrow \uparrow$ \\
\hline Lack of energy & $x x x$ & & & & & & & & & $\downarrow$ & $x$ & - & $x$ & $\uparrow$ & $x$ & ?x \\
\hline Physical symptoms & $x x x$ & & & & & & & & & $x$ & $x$ & - & $x$ & $\uparrow$ & $x$ & $? \uparrow$ \\
\hline Injury & $x x x$ & & & & & & & & & $x$ & $\uparrow$ & $x$ & $\uparrow$ & $\uparrow$ & $x$ & $? \uparrow$ \\
\hline Emotional exhaustion & $x x x$ & & & & & & & & & $\mathrm{x}$ & $\mathrm{x}$ & - & $x$ & $x$ & - & $\uparrow \uparrow$ \\
\hline Disturbed breaks & $x x x$ & & & & & & & & & $x$ & $x$ & - & $x$ & $x$ & - & $?$ \\
\hline Recovery & $x x x$ & & & & & & & & & $\uparrow$ & $x$ & - & $x$ & $\downarrow$ & $x$ & $?$ \\
\hline Social recovery & $x x x$ & & & & & & & & & $x$ & $x$ & - & $x$ & $x$ & - & $x x$ \\
\hline Physical recovery & $x x x$ & & & & & & & & & $\uparrow$ & $x$ & - & $x$ & $\downarrow$ & $x$ & $? \downarrow$ \\
\hline General well-being & $x x x$ & & & & & & & & & $\uparrow$ & $x$ & - & $x$ & $\downarrow$ & $x$ & $? \downarrow$ \\
\hline Sleep quality & $x x x$ & & & & & & & & & $x$ & $x$ & - & $x$ & $x$ & - & $?$ \\
\hline Success & $x x x$ & & & & & & & & & $\uparrow$ & $x$ & - & $x$ & $x$ & - & $x x$ \\
\hline Being in shape & $x x x$ & & & & & & & & & $\uparrow$ & $\uparrow$ & $x$ & $\downarrow$ & $\downarrow$ & $x$ & $? \downarrow$ \\
\hline Personal accomplishment & $x x x$ & & & & & & & & & $x$ & $x$ & - & $x$ & $x$ & - & $x x$ \\
\hline Self-efficacy & $x x x$ & & & & & & & & & $x$ & $x$ & - & $x$ & $x$ & - & $?$ \\
\hline Self-regulation & $?$ & & & & & & & & & $\uparrow$ & $?$ & $x$ & $?$ & $x$ & - & $x x x$ \\
\hline \multicolumn{17}{|l|}{ DALDA } \\
\hline Sources of stress & - & - & & & $x$ & & & & & & & & & - & & $\mathrm{x}$ \\
\hline Symptoms of stress & $x$ & $x$ & & & $\uparrow$ & & & & & & & & & $x$ & & $\uparrow$ \\
\hline \multicolumn{17}{|l|}{ STAI } \\
\hline State anxiety & & $x$ & & & & & & & & & & & & & & \\
\hline PSS & & & & $x$ & $\uparrow$ & $x$ & $x$ & $x$ & $\uparrow$ & & & & & & & \\
\hline \multicolumn{17}{|l|}{ MTDS } \\
\hline Total score & & & & $\mathrm{x}$ & $\mathrm{x}$ & $x$ & $x$ & $\mathrm{x}$ & $\mathrm{x}$ & & & & & & & \\
\hline Depressed mood & & & & $\uparrow$ & $\uparrow$ & $x$ & $\downarrow$ & $x$ & $\uparrow$ & & & & & & & \\
\hline Perceived vigour & & & & $x$ & $\downarrow$ & $x$ & $\uparrow$ & $x$ & $\downarrow$ & & & & & & & \\
\hline Physical symptoms & & & & $x$ & $\downarrow$ & $x$ & $\uparrow$ & $x$ & $x$ & & & & & & & \\
\hline Sleep disturbance & & & & $\downarrow$ & $\uparrow$ & $x$ & $\downarrow$ & $x$ & $\uparrow$ & & & & & & & \\
\hline Perceived stress & & & & $\uparrow$ & $x$ & $\uparrow$ & & $x$ & $\uparrow$ & & & & & & & \\
\hline General fatigue & & & & $x$ & $\uparrow$ & $\mathrm{x}$ & $\downarrow$ & $\uparrow$ & $\uparrow$ & & & & & & & \\
\hline \multicolumn{17}{|l|}{ Mood } \\
\hline All subscales & & - & & & & & & & & & & & & & & \\
\hline $\mathrm{n}$ & 6 & 7 & 2 & 2 & 4 & 2 & 2 & 2 & 2 & 1 & 3 & 2 & 2 & 4 & 2 & 10 \\
\hline
\end{tabular}

Associations rated as positive $(\uparrow)$, negative $(\downarrow)$, not associated $(x)$, conflicting findings (?), no change (-). Three symbols indicates strong evidence, two symbols for moderate evidence and one symbol for limited evidence. Shading highlights a moderate association. Abbreviations for objective measures: leukocytes (Leuk), immunoglobulin-A (IgA), immunoglobulin- $G$ $(\mathrm{IgG})$, interleukins (IL-), tumour necrosis factor $\alpha$ (TNF- $\alpha$ ), C reactive protein (CRP), oxidative stress (Ox. stress), reduced-oxidised glutathione ratio (GSH:GSSG), creatine kinase (CK). $n$ refers to number of contributing studies.

POMS, Profile of Mood States; RESTQ-S, Recovery Stress Questionnaire for Athletes; DALDA, Daily Analyses of Life Demands of Athletes; STAI, State-Trait Anxiety Inventory; PSS,

Perceived Stress Scale; MTDS, Multi-Component Training Distress Scale.

Athlete monitoring is not limited to either subjective or objective measures, instead they can be used to complement each other. Combining both types of measures is common in applied settings, as evident by the large number of studies included in this review and a recent survey of high performance sports. ${ }^{85}$ This is also consistent with recent recommendations. ${ }^{2}{ }^{86}$ 
Table 5 Evidence of associations between subjective and objective (physiological and performance) measures

\begin{tabular}{|c|c|c|c|c|c|c|c|c|c|c|c|c|}
\hline & \multicolumn{10}{|c|}{ Physiological } & \multicolumn{2}{|c|}{ Performance } \\
\hline & La submax & La max & HR rest & HRV & HR submax & HR max & HR rec & $\mathrm{BP}$ rest & $\mathrm{vO}_{2}$ submax & $\mathrm{VO}_{2} \max$ & Sust. & Short \\
\hline \multicolumn{13}{|l|}{ POMS } \\
\hline Total mood disturbance & - & $\mathrm{x}$ & $\mathrm{x}$ & $\uparrow$ & $x x$ & $?$ & & $x$ & $x x x$ & $\downarrow \downarrow \downarrow$ & $?$ & $x x$ \\
\hline Tension & $x$ & $x$ & $\downarrow$ & $x$ & $x x x$ & $\downarrow$ & & $x$ & $x$ & $?$ & $x x$ & $?$ \\
\hline Depression & - & - & $\downarrow$ & $x$ & $x x$ & $x$ & & $x$ & - & $x x x$ & $x x$ & $?$ \\
\hline Anger & - & - & $\downarrow$ & $x$ & $x x$ & $x$ & & $x$ & - & $x x x$ & $x x$ & $x x$ \\
\hline Confusion & $x$ & $x$ & $\downarrow$ & $x$ & $x x x$ & $\downarrow$ & & $x$ & $x$ & $?$ & $?$ & $x x$ \\
\hline Vigour & $x$ & $x x x$ & $x$ & $x$ & $x x$ & $?$ & & $x$ & $x x x$ & $\uparrow \uparrow \uparrow$ & $?$ & $x x x$ \\
\hline Fatigue & $x$ & $x x x$ & ?x & $\uparrow$ & $x x$ & $?$ & & $x$ & $x x x$ & $\downarrow \downarrow$ & $?$ & $x x$ \\
\hline \multicolumn{13}{|l|}{ RESTQ-S } \\
\hline Recovery-Stress Index & & & & & - & - & & & & & $x x$ & $x x x$ \\
\hline Stress & & $x$ & & & $x$ & - & & & & & $x x x$ & $x x$ \\
\hline General stress & & $x$ & & & $x$ & & & & & & $x x x$ & $\uparrow$ \\
\hline Emotional stress & & $x$ & & & $x$ & & & & & & $x x x$ & $\uparrow$ \\
\hline Social stress & & $x$ & & & $x$ & & & & & & $x x x$ & $\uparrow$ \\
\hline Conflicts/pressure & & $x$ & & & $x$ & & & & & & $x x x$ & $?$ \\
\hline Fatigue & & - & & & - & & & & & & $?$ & $x x x$ \\
\hline Lack of energy & & $x$ & & & $x$ & & & & & & $x x$ & $?$ \\
\hline Physical symptoms & & $x$ & & & $x$ & & & & & & $x x$ & $\uparrow$ \\
\hline Injury & & $x$ & & & $x$ & & & & & & $x x$ & $?$ \\
\hline Emotional exhaustion & & $x$ & & & $x$ & & & & & & $x x x$ & $\uparrow$ \\
\hline Disturbed breaks & & $x$ & & & $x$ & & & & & & $x x x$ & $?$ \\
\hline Recovery & & $x$ & & & $x$ & - & & & & & $x x x$ & $x x$ \\
\hline Social recovery & & $x$ & & & $x$ & & & & & & $\uparrow \uparrow$ & $x$ \\
\hline Physical recovery & & - & & & - & & & & & & $? \mathrm{x}$ & $x x x$ \\
\hline General well-being & & $x$ & & & $x$ & & & & & & $x x$ & $?$ \\
\hline Sleep quality & & $x$ & & & $x$ & & & & & & $x x$ & $?$ \\
\hline Success & & - & & & - & & & & & & $x x$ & $x x x$ \\
\hline Being in shape & & - & & & - & & & & & & $?$ & $x x x$ \\
\hline Personal accomplishment & & $x$ & & & $x$ & & & & & & $x x x$ & $\downarrow$ \\
\hline Self-efficacy & & $x$ & & & $x$ & & & & & & $? \mathrm{x}$ & $?$ \\
\hline Self-regulation & & $x$ & & & $x$ & & & & & & $x x x$ & $?$ \\
\hline \multicolumn{13}{|l|}{ SFMS } \\
\hline Overtraining score & $\downarrow$ & $\downarrow$ & & $\uparrow$ & & $\downarrow$ & & & & & $\downarrow$ & $x$ \\
\hline \multicolumn{13}{|l|}{ DALDA } \\
\hline Sources of stress & - & - & - & & - & $x$ & $x$ & & - & $x$ & $x x x$ & $x$ \\
\hline Symptoms of stress & $x \mathrm{xx}$ & $x x x$ & $x$ & & $x x x$ & $?$ & $\uparrow$ & & $x$ & $\downarrow$ & $\downarrow \downarrow$ & $\downarrow$ \\
\hline \multicolumn{13}{|l|}{ CSAI-2 } \\
\hline Cognitive anxiety & & & & & & & & & & - & $x$ & $x$ \\
\hline Somatic anxiety & & & & & & & & & & $x$ & $x$ & $x$ \\
\hline Self-confidence & & & & & & & & & & $x$ & $x$ & $x$ \\
\hline \multicolumn{13}{|l|}{ MTDS } \\
\hline Total score & & & & & & & & & & & $\uparrow$ & \\
\hline DSC & & & & & & & & & & - & $x$ & $x$ \\
\hline \multicolumn{13}{|l|}{ STPI } \\
\hline Anxiety & & & & & & & & & & $x$ & $x$ & $x$ \\
\hline Anger & & & & & & & & & & - & $x$ & $x$ \\
\hline $\mathrm{n}$ & 3 & 6 & 4 & 2 & 9 & 7 & 1 & 1 & 4 & 5 & 29 & 16 \\
\hline
\end{tabular}

\section{Use of subjective measures in practice}

The potential efficacy of subjective measures for athlete monitoring has been established, however optimal implementation practices are yet to be determined. Implementation practices affect how well athlete well-being is reflected, and whether the data can be used in a meaningful way. ${ }^{87}$ The differing practices among the included studies lends to discussion regarding the particular self-report measure used to assess subjective wellbeing, the response set and rating scales and the timing and frequency of administration. 
Table 6 Typical patterns of objective measures to acute changes in training load and chronic training

\begin{tabular}{|c|c|c|c|c|}
\hline & \multicolumn{2}{|l|}{ Acute } & \multirow{2}{*}{$\frac{\text { Chronic }}{\text { Ongoing training }}$} & \multirow[b]{2}{*}{ n } \\
\hline & Increased training load & Decreased training load & & \\
\hline \multicolumn{5}{|l|}{ Endocrine } \\
\hline C & --- & ? & ? & 23 \\
\hline $\mathrm{T}$ & -- & $?$ & $?$ & 10 \\
\hline $\mathrm{T}: \mathrm{C}$ & --- & --- & -- & 8 \\
\hline E/NE & --- & --- & $\downarrow \downarrow \downarrow$ & 6 \\
\hline Prolactin & - & - & - & 2 \\
\hline $\mathrm{GH}$ & & - & - & 1 \\
\hline ACTH & - & - & & 1 \\
\hline LH & & & - & 1 \\
\hline Dopamine & - & - & & 1 \\
\hline IGF-1 & & & & 0 \\
\hline \multicolumn{5}{|l|}{ Erythrocytes } \\
\hline $\mathrm{RBC}$ & & & --- & 2 \\
\hline Hct & - & - & --- & 3 \\
\hline $\mathrm{Hb}$ & $\downarrow \downarrow \downarrow$ & $\uparrow$ & --- & 4 \\
\hline \multicolumn{5}{|l|}{ Immune } \\
\hline Leuk. & -- & -- & $\downarrow \downarrow \downarrow$ & 6 \\
\hline $\lg A$ & --- & --- & $\uparrow \uparrow$ & 7 \\
\hline $\lg G$ & $?$ & - & & 2 \\
\hline IL-1 $\beta$ & & - & --- & 2 \\
\hline IL-6 & & $?$ & --- & 3 \\
\hline IL-8 & & - & --- & 2 \\
\hline IL-10 & & $\uparrow$ & $\downarrow$ & 2 \\
\hline IL-12p70 & & $\downarrow$ & $\uparrow$ & 2 \\
\hline TNF- $\alpha$ & & $\uparrow$ & $\downarrow$ & 2 \\
\hline$\alpha$-amylase & & & $\uparrow$ & 1 \\
\hline \multicolumn{5}{|c|}{ Inflammation an muscle damage } \\
\hline CRP & & & $\downarrow$ & 2 \\
\hline Oxidative stress & & & $?$ & 2 \\
\hline GSH:GSSG & & & $\downarrow$ & 2 \\
\hline Urea & - & - & - & 3 \\
\hline Creatinine & - & - & $\downarrow$ & 2 \\
\hline CK & $\downarrow \downarrow$ & $\uparrow \uparrow$ & --- & 10 \\
\hline \multicolumn{5}{|l|}{ Physiological } \\
\hline La submax & -- & -- & & 3 \\
\hline La max & --- & --- & --- & 6 \\
\hline $\mathrm{HR}_{\text {rest }}$ & - & $?-$ & --- & 4 \\
\hline$H R_{\text {variability }}$ & & & $? \uparrow$ & 2 \\
\hline $\mathrm{HR}_{\text {submax }}$ & --- & -- & --- & 9 \\
\hline $\mathrm{HR}_{\max }$ & -- & -- & --- & 7 \\
\hline$H R_{\text {recovery }}$ & & & $\uparrow$ & 1 \\
\hline $\mathrm{BP}_{\text {rest }}$ & & - & & 1 \\
\hline $\mathrm{VO}_{2}$ submax & --- & --- & $\downarrow$ & 4 \\
\hline $\mathrm{VO}_{2} \max$ & - & $\uparrow \uparrow$ & --- & 5 \\
\hline \multicolumn{5}{|l|}{ Performance } \\
\hline Sustained & $\downarrow \downarrow$ & $\uparrow \uparrow$ & $\uparrow \uparrow$ & 28 \\
\hline Short & $\downarrow \downarrow$ & $\uparrow \uparrow$ & $\uparrow \uparrow$ & 25 \\
\hline
\end{tabular}

Self-report measures may be characterised by: (1) whether or not they are specifically designed for athletes, (2) if they evaluate single or multiple constructs and (3) whether the constructs are based on stressors, or resulting symptoms. It has been suggested that athlete-specific measures evaluating multiple constructs may better reflect performance capacities. ${ }^{88}$ Broader measures may also cater to the differing circumstances and responses of individuals, capturing both training and non-training stressors and 
Table 7 Typical patterns of subjective measures to acute changes in training load and chronic training

\begin{tabular}{|c|c|c|c|c|}
\hline & \multicolumn{2}{|l|}{ Acute } & \multirow{2}{*}{$\frac{\text { Chronic }}{\text { Ongoing training }}$} & \multirow[b]{2}{*}{$\mathrm{n}$} \\
\hline & Increased training load & Decreased training load & & \\
\hline \multicolumn{5}{|l|}{ POMS } \\
\hline Total mood disturbance & $\downarrow \downarrow$ & $\uparrow \uparrow \uparrow$ & $?$ & 27 \\
\hline Tension & $?$ & $\uparrow \uparrow$ & - & 27 \\
\hline Depression & $?-$ & -- & -- & 28 \\
\hline Anger & $?$ & $\uparrow \uparrow$ & $?$ & 28 \\
\hline Confusion & -- & -- & --- & 27 \\
\hline Vigour & $\downarrow \downarrow \downarrow$ & $\uparrow \uparrow$ & $?$ & 29 \\
\hline Fatigue & $\downarrow \downarrow \downarrow$ & $\uparrow \uparrow \uparrow$ & $?$ & 30 \\
\hline Recovery-Stress Index & $\downarrow \downarrow$ & $\uparrow \uparrow \uparrow$ & $\downarrow$ & 7 \\
\hline Stress & $\downarrow \downarrow$ & $\uparrow \uparrow \uparrow$ & $\downarrow \downarrow$ & 10 \\
\hline General stress & -- & $\uparrow \uparrow$ & $\downarrow \downarrow \downarrow$ & 11 \\
\hline Emotional stress & -- & $?$ & -- & 11 \\
\hline Social stress & -- & $\uparrow \uparrow$ & $\downarrow \downarrow$ & 11 \\
\hline Conflicts/pressure & --- & --- & $\downarrow \downarrow \downarrow$ & 11 \\
\hline Fatigue & $\downarrow \downarrow \downarrow$ & $\uparrow \uparrow$ & $\downarrow \downarrow$ & 11 \\
\hline Lack of energy & --- & -- & $\downarrow \downarrow \downarrow$ & 11 \\
\hline Physical symptoms & $\downarrow \downarrow$ & $\uparrow \uparrow$ & -- & 11 \\
\hline Injury & $\downarrow \downarrow$ & $\uparrow \uparrow \uparrow$ & ?- & 11 \\
\hline Emotional exhaustion & -- & -- & -- & 11 \\
\hline Disturbed breaks & --- & -- & -- & 11 \\
\hline Recovery & $\downarrow \downarrow$ & $\uparrow \uparrow$ & $\downarrow \downarrow$ & 10 \\
\hline Social recovery & -- & --- & --- & 11 \\
\hline Physical recovery & $\downarrow \downarrow$ & $\uparrow \uparrow \uparrow$ & $\downarrow \downarrow$ & 11 \\
\hline General well-being & $\downarrow \downarrow$ & $\uparrow \uparrow \uparrow$ & $\downarrow \downarrow \downarrow$ & 11 \\
\hline Sleep quality & --- & -- & -- & 11 \\
\hline Success & --- & --- & $\downarrow \downarrow$ & 11 \\
\hline Being in shape & $\downarrow \downarrow \downarrow$ & $\uparrow \uparrow \uparrow$ & $\downarrow \downarrow$ & 11 \\
\hline Personal accomplishment & --- & --- & $\downarrow \downarrow$ & 11 \\
\hline Self-efficacy & -- & -- & -- & 11 \\
\hline Self-regulation & --- & --- & $\downarrow \downarrow \downarrow$ & 11 \\
\hline \multicolumn{5}{|l|}{ SFMS } \\
\hline Overtraining score & $\downarrow$ & $\uparrow$ & -- & 4 \\
\hline \multicolumn{5}{|l|}{ DALDA } \\
\hline Sources of stress & --- & --- & - & 4 \\
\hline Symptoms of stress & $\downarrow \downarrow \downarrow$ & $\uparrow \uparrow \uparrow$ & $\downarrow$ & 5 \\
\hline \multicolumn{5}{|l|}{ STAI } \\
\hline State anxiety & & & - & 1 \\
\hline \multicolumn{5}{|l|}{ CSAI-2 } \\
\hline Cognitive anxiety & & & - & 1 \\
\hline Somatic anxiety & & & $?$ & 1 \\
\hline Self-confidence & & & $?$ & 1 \\
\hline PSS & $?$ & $\uparrow$ & & 1 \\
\hline \multicolumn{5}{|l|}{ MTDS } \\
\hline Total score & & & $\downarrow$ & 1 \\
\hline All subscales & & & & 0 \\
\hline DSC & & & - & 1 \\
\hline \multicolumn{5}{|l|}{ STPI } \\
\hline Anxiety & & & $?$ & 1 \\
\hline Anger & & & - & 1 \\
\hline \multicolumn{5}{|l|}{ Mood } \\
\hline All subscales & & & - & 1 \\
\hline
\end{tabular}


their systemic influence on subjective well-being. ${ }^{3}$ Therefore, on balance, the RESTQ- ${ }^{38}$ and MTDS $^{43}$ are more promising selfreport measures.

We found that the RESTQ-S was the only investigated self-report measure to be responsive to both acute and chronic training load. One stress (fatigue) and three recovery (physical recovery, general well-being, being in shape) subscales responded to both acute and chronic training. Interestingly, while a larger number of subscales were less responsive or unresponsive, the overall stress and recovery scores were shown to be responsive across training conditions. This is in contrast to the observation that consolidation of subscales into a total score typically reduced sensitivity. Nevertheless, the non-uniform responsiveness of subscales within a measure illustrates the need to consider subscales on an individual basis.

The MTDS is a more recently developed and hence less widely investigated self-report measure. The strength of the MTDS over the RESTQ-S is the inclusion of mood disturbance, stress and behavioural symptom subscales with a smaller number of items (22 compared to 76 for the RESTQ-S). Breadth and a small number of items is a key consideration to sustain use of self-report measures in practice. ${ }^{89}$ These two issues are often the reason that sports programmes tend to incorporate elements of established measures into their own brief custom self-report measure rather than adopting an existing measure from the literature. ${ }^{85}$ Implications of this review for practice should therefore be for sports programmes to consider which independent subscales may offer greater utility for monitoring athlete well-being.

To monitor acute changes in athlete well-being, the following subscales may be useful: vigour/motivation, physical symptoms/ injury, non-training stress, fatigue, physical recovery, general health/well-being, and being in shape. These subscales provide the practitioner with insight into the athlete's ability to perform training that day, so may be useful for guiding adjustments to prescribed training on an individual basis. These subscales are consistent with previous recommendations. ${ }^{148}$ Previous recommendations have also included irritability, sleep, willingness to train, enjoyment of training and how training felt, which correspond to symptoms of stress as recorded by the DALDA; ${ }^{39}$ which may also be useful for monitoring acute well-being.

For ongoing monitoring, utility was demonstrated in five of the subscales which responded acutely (non-training stress, fatigue, physical recovery, general health/well-being, being in shape). Three other subscales (conflicts/pressure, self-regulation, lack of energy) also presented utility for monitoring chronic well-being, however it is unclear whether their inclusion would offer any additional benefit beyond the previously mentioned subscales.

Importantly, we also identified subscales that were unresponsive (eg, depression, confusion, emotional stress, social recovery, sleep quality, self-efficacy). These may not be useful for monitoring athlete well-being. There appeared to be no value in quantifying non-training stressors, instead it is their effect on athlete well-being that is most meaningful. Interestingly, the depression subscale (POMS) was unresponsive despite depression being a symptom of overtraining syndrome. ${ }^{5} 20$ This may be attributable to different patterns of mood disturbance between overreached and overtrained athletes, with depression only increasing in overtraining syndrome. ${ }^{90}$ Therefore, the inclusion of this subscale may be valuable if an athlete were to progress to overtraining syndrome.

The response sets used by reviewed studies typically utilised the standard questionnaire response set (eg, POMS in the past week', RESTQ-S 'in the past 3 days/nights'), which assessed a relatively transient state of acute well-being. This was the case regardless of time between measurements. Capturing transient well-being is important, particularly during acute changes in training load, ${ }^{3}$ as relevant changes may be diluted or compromised by recall error if athletes are asked to summarise a period too long in the past. $^{91}$ Typically subjective measures utilise Likert scales (eg, never (0) to always (6) as in the RESTQ-S), yet their use in the applied setting requires repeated measurement to establish an individual's baseline from which changes can be determined. This may be avoided by having athletes compare their current well-being to normal (ie, worse than normal, normal, better than normal) as in the DALDA.

With regard to the timing and frequency of administration, consideration must be given to minimising the burden on athletes, while obtaining meaningful data that can be acted on if required. Of the reviewed studies, changes in response to acute training loads were typically detected under monitoring on a daily to weekly basis, whereas changes in response to ongoing training were typically monitored at intervals of 1 month or more, either at regular intervals or coinciding with transitions between training phases. In practice, frequent monitoring is needed to enable acute adjustments to training as required. Consequently, previous recommendations for daily monitoring using a measure self-administered by the athlete remain appropriate. $^{1}$ 3-6 8899293 This may be supplemented with a more comprehensive measure such as the POMS or RESTQ-S on a weekly basis, ${ }^{1} 892$ and objective measures on a less-frequent basis such as once per training microcycle. ${ }^{18} 82$

\section{Limitations and strengths}

We included studies with concurrent subjective and objective measure which allowed us to compare the relative responsiveness of subjective and objective measures as they were exposed to the same study conditions. Within the methods of included studies, differing sampling frequencies and response sets is an inherent limitation of this review. Also, while subjective and objective measures had to be concurrent, in most cases athletes were responding retrospectively on a subjective measure (eg, 'in the past week') while the objective measure was of their present well-being. Theoretically these timeframe inconsistencies may have reduced the associations between these two types of measures, and affected the conclusions drawn in this review, but in practice this seems unlikely to explain our overall findings.

An additional limitation when interpreting and comparing study findings is the dependence on the statistical methods used in each study. Most studies used traditional methods ( $\mathrm{p}$ values), however this may have increased the risk of type II error, particularly given small participant numbers. ${ }^{94}$ Some studies may have been underpowered to detect change which would lead us to under-estimate the strength of evidence in our review. Meta-analysis may help to address this limitation in the future. The presentation of group responses may have discounted potentially relevant individual responses. On the other hand, findings may have been overestimated as a result of selective reporting by included studies (publication bias) when there was a lack of change in subjective subscales or objective markers.

\section{SUMMARY}

Subjective well-being responded consistently to stress imposed by training, deteriorating with increased and chronic training and improving with reduced training. There was negligible evidence for an association between subjective and objective measures. This was likely due to superior responsiveness of 
subjective measures over objective measures. Given that subjective measures reflect changes in athlete well-being and provide a practical method for athlete monitoring, coaches and support staff may employ self-report measures with confidence.

\section{What are the findings?}

- Subjective measures respond to training-induced changes in athlete well-being.

- Subjective well-being typically worsened with an acute increase in training load and with a chronic training load; and improved with an acute decrease in training load.

- There was no consistent association between subjective and objective measures.

\section{How might it impact on clinical practice in the future?}

- Subjective measures are useful for athlete monitoring, and practitioners may employ them with confidence.

- Subscales which evaluate non-training stress, fatigue, physical recovery, general health/well-being and being in shape are responsive to both acute and chronic training.

- We recommend that athletes report their subjective well-being on a regular basis and alongside other athlete monitoring practices.

Twitter Follow Anna Saw at @annaesaw

Acknowledgements The authors thank Dr Sam Robertson for providing initial guidance on the search strategy, and Dr Shona Halson for providing feedback on an earlier version of this review.

Funding This research was conducted with support from Deakin University and the Australian Institute of Sport.

Competing interests None declared.

Provenance and peer review Not commissioned; externally peer reviewed.

Open Access This is an Open Access article distributed in accordance with the Creative Commons Attribution Non Commercial (CC BY-NC 4.0) license, which permits others to distribute, remix, adapt, build upon this work non-commercially, and license their derivative works on different terms, provided the original work is properly cited and the use is non-commercial. See: http://creativecommons.org/ licenses/by-nc/4.0/

\section{REFERENCES}

1 Fry RW, Morton AR, Keast D. Overtraining in athletes. Sports Med 1991;12:32-65.

2 Coutts A, Cormack SJ. Monitoring the training response. In: Joyce D, Lewindon D, eds. High-performance training for sports. Champaign, IL: Human Kinetics Publishers, 2014:71-84.

3 Meeusen $\mathrm{R}$, Duclos M, Foster $\mathrm{C}$, et al. Prevention, diagnosis and treatment of the overtraining syndrome: joint consensus statement of the European College of Sport Science (ECSS) and the American College of Sports Medicine (ACSM). Eur J Sport Sci 2013;13:1-24

4 Kuipers H, Keizer H. Overtraining in elite athletes. Sports Med 1988;6:79-92.

5 Armstrong LE, VanHeest JL. The unknown mechanism of the overtraining syndrome: clues from depression and psychoneuroimmunology. Sports Med 2002:32:185-209.

6 Kenttä G, Hassmén P. Overtraining and recovery: a conceptual model. Sports Med 1998:26:1-16.

7 Urhausen A, Kindermann W. Diagnosis of overtraining: what tools do we have? Sports Med 2002:32:95-102.

8 Hooper SL, Mackinnon LT. Monitoring overtraining in athletes. Sports Med $1995 ; 20: 321-7$
9 Smith DJ. A framework for understanding the training process leading to elite performance. Sports Med 2003:33:1103-26.

10 DiFiori JP, Benjamin HJ, Brenner JS, et al. Overuse injuries and burnout in youth sports: a position statement from the American Medical Society for Sports Medicine. Br J Sports Med 2014;48:287-8.

11 Currell K, Jeukendrup AE. Validity, reliability and sensitivity of measures of sporting performance. Sports Med 2008;38:297-316.

12 Hug M, Mullis PE, Vogt $M$, et al. Training modalities: over-reaching and over-training in athletes, including a study of the role of hormones. Best Pract Res Clin Endocrinol Metab 2003;17:191-209.

13 Lehmann M, Foster C, Dickhuth $\mathrm{H}-\mathrm{H}$, et al. Autonomic imbalance hypothesis and overtraining syndrome. Med Sci Sports Exerc 1998;30:1140-5.

14 Petibois C, Cazorla G, Poortmans J-R, et al. Biochemical aspects of overtraining in endurance sports. Sports Med 2003;33:83-94

15 Robson PJ. Elucidating the unexplained underperformance syndrome in endurance athletes: the interleukin-6 hypothesis. Sports Med 2003;33:771-81.

16 Smith LL. Cytokine hypothesis of overtraining: a physiological adaptation to excessive stress? Med Sci Sports Exerc 2000;32:317-31.

17 Urhausen A, Gabriel H, Kindermann W. Blood hormones as markers of training stress and overtraining. Sports Med 1995;20:251-76.

18 Fry AC, Kraemer WJ. Resistance exercise overtraining and overreaching. Sports Med 1997:23:106-29.

19 Lac G, Maso F. Biological markers for the follow-up of athletes throughout the training season. Pathol Biol 2004;52:43-9.

20 Morgan WP, Brown DR, Raglin JS, et al. Psychological monitoring of overtraining and staleness. Br J Sports Med 1987;21:107-14.

21 Cerin E, Szabo A, Hunt N, et al. Temporal patterning of competitive emotions: a critical review. J Sports Sci 2000;18:605-26.

22 Van Tulder M, Furlan A, Bombardier C, et al. Updated method guidelines for systematic reviews in the Cochrane Collaboration Back Review Group. Spine 2003;28:1290-9.

23 Reurink G, Goudswaard GJ, Moen MH, et al. Myotoxicity of injections for acute muscle injuries: a systematic review. Sports Med 2014:44:943-56.

*24 Filaire E, Legrand B, Bret K, et al. Psychobiologic responses to 4 days of increased training and recovery in cyclists. Int J Sports Med 2002;23:588-94.

*25 Filaire E, Legrand B, Lac G, et al. Training of elite cyclists: effects on mood state and selected hormonal responses. J Sports Sci 2004:22:1025-33.

*26 Halson SL, Bridge MW, Meeusen R, et al. Time course of performance changes and fatigue markers during intensified training in trained cyclists. J App/ Physiol 2002;93:947-56.

*27 Halson SL, Lancaster GI, Jeukendrup AE, et al. Immunological responses to overreaching in cyclists. Med Sci Sports Exerc 2003;35:854-61.

28 Brink MS, Visscher C, Coutts AJ, et al. Changes in perceived stress and recovery in overreached young elite soccer players. Scand J Med Sci Sports 2012;22:285-92.

29 Brink MS, Visscher C, Schmikli SL, et al. Is an elevated submaximal heart rate associated with psychomotor slowness in young elite soccer players? Eur J Sport Sci 2013:13:207-14.

30 lellamo F, Pigozzi F, Spataro A, et al. Autonomic and psychological adaptations in Olympic rowers. J Sports Med Phys Fitness 2006;46:598-604.

31 Meister S, Faude 0, Ammann T, et al. Indicators for high physical strain and overload in elite football players. Scand J Med Sci Sports 2013;23:156-63.

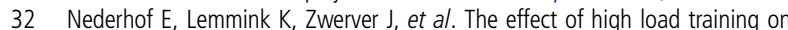
psychomotor speed. Int J Sports Med 2007;28:595-601.

33 Schmitt L, Regnard J, Desmarets $M$, et al. Fatigue shifts and scatters heart rate variability in elite endurance athletes. PLOS ONE 2013;8:e71588.

*34 Chennaoui M, Desgorces F, Drogou C, et al. Effects of Ramadan fasting on physical performance and metabolic, hormonal, and inflammatory parameters in middle-distance runners. App/ Physiol Nutr Metab 2009:34:587-94.

*35 Chtourou H, Hammouda O, Souissi H, et al. The effect of Ramadan fasting on physical performances, mood state and perceived exertion in young footballers. Asian J Sports Med 2011:2:177-85.

36 McNair P, Lorr M, Droppleman L. POMS manual. 2nd edn. San Diego, CA Education and Industrial Testing Service, 1981.

37 Terry PC, Lane AM, Lane HJ, et al. Development and validation of a mood measure for adolescents. J Sports Sci 1999;17:861-72.

38 Kellmann M, Kallus K. Recovery-stress questionnaire for athletes: user manual. Champaign, IL: Human Kinetics, 2001.

39 Rushall BS. A tool for measuring stress tolerance in elite athletes. I App/ Sport Psychol 1990:2:51-66.

40 Legros P. Le surentraînement: diagnostic des manifestations psychocomportementales précoces. Sci Sports 1993;8:71-4.

41 Spielberger CD, Gorsuch RL, Lushene RE. Manual for the state-trait anxiety inventory. Palo Alto, CA: Consulting Psychologists Press, 1970

42 Cohen S, Kamarck T, Mermelstein R. A global measure of perceived stress. $J$ Health Soc Behav 1983;24:385-96.

43 Main L, Grove JR. A multi-component assessment model for monitoring training distress among athletes. Eur J Sport Sci 2009;9:195-202. 
44 Martens R, Vealey RS, Burton D, et al. Development and validation of the competitive sports anxiety inventory 2. In: Martens R, Vealey RS, Burton D, eds. Competitive anxiety in sport. Champaign, IL: Human kinetics, 1990: $117-78$.

45 Derogatis LR. SCL-90: administration, scoring and procedures manual for the $R$ (evised) version. Baltimore, MD: Johns Hopluns University School of Medicine, 1977.

46 Spielberger CD, Barker LR, Russell SF, et al. The preliminary manual for the State-Trait Personality Inventory [Unpublished manual]. Tampa: University of South Florida, 1979.

47 Choi PY, Salmon P. Symptom changes across the menstrual cycle in competitive sportswomen, exercisers and sedentary women. Br J Clin Psychol 1995;34:447-60.

*48 Coutts AJ, Wallace LK, Slattery KM. Monitoring changes in performance, physiology, biochemistry, and psychology during overreaching and recovery in triathletes. Int J Sports Med 2007;28:125-34.

*49 Mäestu J, Jürimäe J, Kreegipuu K, et al. Changes in perceived stress and recovery during heavy training in highly trained male rowers. Sport Psychol 2006;20:24-39.

*50 Jürimäe J, Mäestu J, Purge $P$, et al. Changes in stress and recovery after heavy training in rowers. J Sci Med Sport 2004;7:335-9.

*51 Jürimäe J, Mäestu J, Purge $P$, et al. Relations among heavy training stress, mood state, and performance for male junior rowers. Percept Mot Skills 2002;95:520-6.

*52 Coutts AJ, Reaburn P. Monitoring changes in rugby league players' perceived stress and recovery during intensified training. Percept Mot Skills 2008; 106:904-16.

*53 Kellmann M, Altenburg D, Lormes W, et al. Assessing stress and recovery during preparation for the World Championships in rowing. Sport Psychol 2001; 15:151-67.

*54 Martin DT, Andersen MB, Gates W. Using Profile of Mood States (POMS) to monitor high-intensity training in cyclists: group versus case studies. Sport Psychol 2000;14:138-56.

*55 Verde T, Thomas S, Shephard RJ. Potential markers of heavy training in highly trained distance runners. Br J Sports Med 1992;26:167-75.

*56 Main LC, Dawson B, Grove JR, et al. Impact of training on changes in perceived stress and cytokine production. Res Sports Med 2009;17: 121-32.

*57 Coutts AJ, Slattery KM, Wallace LK. Practical tests for monitoring performance, fatigue and recovery in triathletes. J Sci Med Sport 2007;10:372-81.

*58 Berger BG, Motl RW, Butki BD, et al. Mood and cycling performance in response to three weeks of high-intensity, short-duration overtraining, and a two-week taper. Sport Psychol 1999;13:444-57.

*59 Bosquet L, Léger L, Legros P. Blood lactate response to overtraining in male endurance athletes. Eur J App/ Physiol 2001;84:107-14.

*60 Robson-Ansley PJ, Blannin A, Gleeson M. Elevated plasma interleukin-6 levels in trained male triathletes following an acute period of intense interval training. Eur J App/ Physiol 2007;99:353-60.

*61 Umeda T, Suzukawa K, Takahashi I, et al. Effects of intense exercise on the physiological and mental condition of female university judoists during a training camp. J Sports Sci 2008;26:897-904.

*62 O'Connor PJ, Morgan WP, Raglin JS. Psychobiologic effects of $3 \mathrm{~d}$ of increased training in female and male swimmers. Med Sci Sports Exerc 1991;23: 1055-61.

*63 Raglin JS, Koceja DM, Stager JM, et al. Mood, neuromuscular function, and performance during training in female swimmers. Med Sci Sports Exerc 1996;28:372-7.

*64 O'Connor PJ, Morgan WP, Raglin JS, et al. Mood state and salivary cortisol levels following overtraining in female swimmers. Psychoneuroendocrinology 1989;14:303-10.

*65 Hooper SL, Mackinnon LT, Ginn EM. Effects of three tapering techniques on the performance, forces and psychometric measures of competitive swimmers. Eur J Appl Physiol Occup Physiol 1998;78:258-63.

*66 Koutedakis Y, Budgett R, Faulmann L. Rest in underperforming elite competitors. Br J Sports Med 1990;24:248-52.

*67 O'Connor PJ, Morgan WP, Koltyn KF, et al. Air travel across four time zones in college swimmers. J App/ Physiol 1991;70:756-63.

68 Kazdin AE. Reactive self-monitoring: the effects of response desirability, goal setting, and feedback. J Consult Clin Psychol 1974;42:704-16.

*69 Faude 0, Kellmann M, Ammann T, et al. Seasonal changes in stress indicators in high level football. Int J Sports Med 2011;32:259-65.

*70 Filaire E, Ferreira JP, Oliveira M, et al. Diurnal patterns of salivary alpha-amylase and cortisol secretion in female adolescent tennis players after 16 weeks of training. Psychoneuroendocrinology 2013;38:1122-32.

*71 Filaire E, Rouveix M, Duclos M. Training and 24-hr urinary catecholamine excretion. Int J Sports Med 2009;30:33-9.

*72 Dodson DL. Over-training syndrome: a study to determine the correlation between the physiological symptoms and the psychological signs in college wrestlers. ProQuest Information \& Learning, 2007.
*73 Filaire E, Bernain X, Sagnol M, et al. Preliminary results on mood state, salivary testosterone:cortisol ratio and team performance in a professional soccer team. Eur J App/ Physiol 2001;86:179-84.

*74 Rouveix M, Duclos M, Gouarne C, et al. The $24 \mathrm{~h}$ urinary cortisol/cortisone ratio and epinephrine/norepinephrine ratio for monitoring training in young female tennis players. Int J Sports Med 2006;27:856-63.

*75 Kumae T, Suzukawa K, Ishii T. Effects of 6 months of endurance training on neutrophil functions to produce reactive oxygen species and mental states in male long-distance runners. Luminescence 2013;28:23-9.

*76 Elloumi M, Ounis OB, Tabka Z, et al. Psychoendocrine and physical performance responses in male Tunisian rugby players during an international competitive season. Aggress Behav 2008;34:623-32.

*77 Rama L, Alves F, Teixeira A. Hormonal, immune, autonomic and mood state variation in the initial preparation phase of a winter season, in Portuguese male swimmers. Xlth International Symposium for Biomechanics and Medicine in Swimming. 2010:217-19.

*78 Liederbach M, Gleim GW, Nicholas JA. Monitoring training status in professional ballet dancers. J Sports Med Phys Fitness 1992;32:187-95.

79 Hooper SL, Mackinnon LT, Howard A. Markers for monitoring overtraining and recovery. Med Sci Sports Exerc 1995;27:106-12.

80 Brancaccio P, Maffulli N, Limongelli FM. Creatine kinase monitoring in sport medicine. Br Med Bull 2007;81-82:209-30.

81 Jokela M, Hanin YL. Does the individual zones of optimal functioning model discriminate between successful and less successful athletes? A meta-analysis. J Sports Sci 1999;17:873-87.

82 Halson SL, Jeukendrup AE. Does overtraining exist? An analysis of overreaching and overtraining research. Sports Med 2004;34:967-81.

83 Reid VL, Gleeson M, Williams N, et al. Clinical investigation of athletes with persistent fatigue and/or recurrent infections. Br J Sports Med 2004;38:42-5.

84 Burden RJ, Morton K, Richards T, et al. Is iron treatment beneficial in, iron-deficient but non-anaemic (IDNA) endurance athletes? A meta-analysis. Br J Sports Med 2015:49:1389-97.

85 Taylor K, Chapman D, Cronin J, et al. Fatigue monitoring in high performance sport: a survey of current trends. J Aust Strength Cond 2012;20:12-23.

86 Halson SL. Monitoring training load to understand fatigue in athletes. Sports Med 2014;44(S2):139-47.

87 Saw AE, Main LC, Gastin PB. Monitoring athletes through self-report: factors influencing implementation. J Sports Sci Med 2015;14:137-46.

88 Grove JR, Main LC, Partridge K, et al. Training distress and performance readiness: laboratory and field validation of a brief self-report measure. Scand J Med Sci Sports 2014;24:e483-90.

89 Gastin PB, Meyer D, Robinson D. Perceptions of wellness to monitor adaptive responses to training and competition in elite Australian football. J Strength Cond Res 2013;27:2518-26.

90 Raglin JS, Morgan WP. Development of a scale for use in monitoring training-induced distress in athletes. Int J Sports Med 1994;15:84-8.

91 Shiffman $S$. Real-time self-report of momentary states in the natural environment: computerized ecological momentary assessment. In: Stone AA, Turkkan JS, Bachrach CA, et al, eds. The science of self-report: implications for research and practice. Mahwah, NJ: Lawrence Erlbaum Associates, 2000:277-96.

92 Botterill C, Wilson C. Overtraining: emotional and interdisciplinary dimensions. In: Kellman $\mathrm{M}$, ed. Enhancing recovery: preventing underperformance in athletes. Champaign, IL: Human Kinetics, 2002:143-59.

93 Kellmann M, Patrick T, Botterill C, et al. The recovery-cue and its use in applied settings: practical suggestions regarding assessment and monitoring of recovery. In: Kellmann M, ed. Enhancing recovery: preventing underperformance in athletes. Champaign, IL: Human Kinetics, 2002:219-29.

94 Batterham AM, Hopkins WG. Making meaningful inferences about magnitudes. Int J Sports Physiol Perform 2006;1:50-7.

*95 Bresciani G, Cuevas MJ, Garatachea N, et al. Monitoring biological and psychological measures throughout an entire season in male handball players. Eur J Sport Sci 2010;10:377-84.

*96 Burrows M, Bird SR, Bishop N. The menstrual cycle and its effect on the immune status of female endurance runners. J Sports Sci 2002;20:339-44.

*97 Filaire E, Bonis J, Lac G. Relationships between physiological and psychological stress and salivary immunoglobulin A among young female gymnasts. Percept Mot Skills 2004;99:605-17.

*98 Garatachea N, García-López D, José Cuevas M, et al. Biological and psychological monitoring of training status during an entire season in top kayakers. J Sports Med Phys Fitness 2011;51:339-46.

*99 Gleeson M, McDonald WA, Pyne DB, et al. Salivary IgA levels and infection risk in elite swimmers. Med Sci Sports Exerc 1999:31:67-73.

*100 González-Bono E, Salvador A, Serrano MA, et al. Effects of training volume on hormones and mood in basketball players. Int J Stress Manag 2002;9:263-73.

*101 Jones MT, Matthews TD, Murray M, et al. Psychological correlates of performance in female athletes during a 12-week off-season strength and conditioning program. J Strength Cond Res 2010;24:619-28. 
*102 Kenttä G, Hassmén P, Raglin JS. Mood state monitoring of training and recovery in elite kayakers. Eur J Sport Sci 2006;6:245-53.

*103 Lamberts RP, Rietjens GJ, Tijdink HH, et al. Measuring submaximal performance parameters to monitor fatigue and predict cycling performance: a case study of a world-class cyclo-cross cyclist. Eur J Appl Physiol 2010;108:183-90.

*104 Leti T, Bricout VA. Interest of analyses of heart rate variability in the prevention of fatigue states in senior runners. Auton Neurosci 2013;173:14-21.

*105 Main LC, Dawson B, Heel K, et al. Relationship between inflammatory cytokines and self-report measures of training overload. Res Sports Med 2010;18:127-39.

*106 Milanez VF, Ramos SP, Okuno NM, et al. Evidence of a non-linear dose-response relationship between training load and stress markers in elite female futsal players. J Sports Sci Med 2014;13:22-9.

*107 Murphy SM, Fleck SJ, Dudley G, et al. Psychological and performance concomitants of increased volume training in elite athletes. J App/ Sport Psychol 1990;2:34-50.

*108 Papacosta E, Gleeson M, Nassis GP. Salivary hormones, IgA, and performance during intense training and tapering in judo athletes. J Strength Cond Res 2013;27:2569-80.

*109 Purge P, Jürimäe J, Jürimäe T. Hormonal and psychological adaptation in elite male rowers during prolonged training. I Sports Sci 2006;24:1075-82.

*110 Rollo I, Impellizzeri FM, Zago M, et al. Effects of 1 versus 2 games a week on physical and subjective scores of subelite soccer players. Int I Sports Physiol Perform 2014;9:425-31.
*111 Santhiago V, Da Silva ASR, Papoti M, et al. Effects of 14-week swimming training program on the psychological, hormonal, and physiological parameters of elite women athletes. J Strength Cond Res 2011;25:825-32.

*112 Schultz de Arruda AF, Moreira A, Nunes JA, et al. Monitoring stress level of Brazilian female basketball athletes the preparation for the 2009 American Cup. Rev Bras Med Esporte 2013;19:44-7.

*113 Silva ASR, Santhiago V, Papoti M, et al. Psychological, biochemical and physiological responses of Brazilian soccer players during a training program. Sci Sports 2008;23:66-72.

*114 Wallace L, Slattery K, Coutts A. A comparison of methods for quantifying training load: relationships between modelled and actual training responses. Eur J Appl Physiol 2014;114:11-20.

*115 Wittig AF, McConell GK, Costill DL, et al. Psychological effects during reduced training volume and intensity in distance runners. Int I Sports Med 1992;13:497-9.

*116 Wood RE, Hayter S, Rowbottom D, et al. Applying a mathematical model to training adaptation in a distance runner. Eur J Appl Physiol 2005;94: $310-16$.

*Denotes studies included in systematic review and outlined in online supplementary table S1. 